\title{
Clinical and Quantitative MRI Perfusion Analysis of Osteoid Osteomas Before and After Microwave Ablation
}

\author{
MICHAEL KOSTRZEWA, THOMAS HENZLER, STEFAN O. SCHOENBERG, \\ STEFFEN J. DIEHL and NILS RATHMANN
}

\begin{abstract}
Institute of Clinical Radiology and Nuclear Medicine, University Medical Center Mannheim, Medical Faculty Mannheim, University of Heidelberg, Mannheim, Germany
\end{abstract}

\begin{abstract}
Aim: Evaluation of osteoid osteoma (OO) perfusion characteristics pre and post microwave ablation (MWA) using dynamic contrast-enhanced magnetic resonance imaging (DCE-MRI) with clinical follow-up. Materials and Methods: Patients with OO underwent T1weighted 3D DCE-MRI at 3.OT pre and post MWA. Signal intensity (SI) and perfusion of lesions was measured. Patients answered a questionnaire for clinical follow-up. Results: A total of 24 patients were included (mean age of 28 years; nine females); 21 DCE-MRI datasets were of sufficient quality for analysis. The mean SI of lesions, plasma flow, volume of distribution and pain level all decreased statistically significantly after ablation. Conclusion: Quantitative MRI-perfusion analysis allows for objective treatment evaluation after MWA of OO, plasma flow and volume of distribution seem to be reliable indicators for successful thermoablation. MWA is an effective treatment option for $O O$ with a low risk profile and significant pain reduction after treatment.
\end{abstract}

Osteoid osteomas (OO) are small, benign bone tumors which histologically present with a nidus of woven bone containing small capillary channels and peripheral nerve fibers, surrounded by osteoblasts, fibrovascular tissue and reactive sclerosis (1). They cause dull bone pain, which is typically worse at night and can effectively be treated with nonsteroidal anti-inflammatory drugs. The current gold standard of treatment of $\mathrm{OO}$ is percutaneous thermoablation using radiofrequency (2-4). However, it was demonstrated

Correspondence to: Michael Kostrzewa, Theodor-Kutzer-Ufer 1-3, 68167 Mannheim, Germany. Tel: +49 6213832067, Fax: +49 6213831910, e-mail: michael.kostrzewa@medma.uni-heidelberg.de

Key Words: Osteoid osteoma, microwave ablation, perfusion magnetic resonance imaging, dynamic contrast-enhanced magnetic resonance imaging, computed tomography, guidance, minimal invasive. that thermal ablation using microwave ablation (MWA) is an effective treatment alternative (5-7).

For diagnosis of OO, computed tomography (CT) is the imaging modality of choice. Yet radiation dose concerns in the often young patient population necessitate the use of radiation-free imaging modalities. Several studies showed that high-resolution T1-weighted dynamic contrast-enhanced magnetic resonance imaging (DCE-MRI) can also reliably facilitate the diagnosis of OO (8-12). However, data on quantitative DCE-MRI perfusion analysis in patients with $\mathrm{OO}$ treated with microwave ablation are lacking.

Thus, the aim of this prospective study was to quantitatively evaluate $\mathrm{OO}$ perfusion characteristics prior to and after percutaneous MWA using DCE-MRI and to conduct a long-term follow-up using a clinical questionnaire on our patient collective.

\section{Materials and Methods}

The study was approved by the Institutional Review Board (2013814R-MA), and informed consent was obtained from all individual participants (or their parent or legal guardian in the case of children under 16 years of age) included in the study.

All patients who were clinically diagnosed with an $\mathrm{OO}$ by our in-house orthopedic staff underwent T1-weighted 3D DCE-MRI at 3.0T (Magnetom Tim Trio/Skyra; Siemens Healthineers, Erlangen, Germany) before and within 2 days after MWA. Gadolinium chelate Gd-DOTA (0.5 M, Dotarem; Guerbet, Roisy, France) was administered at a dose of $0.03 \mathrm{mmol} / \mathrm{kg}$ with a flow rate of $1.5 \mathrm{ml} / \mathrm{s}$ followed by a $30 \mathrm{ml}$ saline bolus. 3D DCE-MRI was performed using a 3D gradient echo sequence with parallel imaging and stochastic data sampling with time-resolved imaging with stochastic trajectories (TWIST). Parameters were: repetition time/echo time $=2.98 / 1.14(\mathrm{~ms})$, flip angle $=25^{\circ}$, voxel size $=0.94 \mathrm{~mm}^{3}$, parallel imaging $=3$, and temporal resolution $3.8 \mathrm{~s}$, as previously described (13-15)

For all patients, CT-guided MWA was performed under general anesthesia. An initial non-enhanced CT scan was conducted for planning (Somatom Definition/Flash; Siemens Healthineers). Under CT guidance, a 16-gauge ablation needle (Medwaves, San Diego, CA, USA) was introduced centrally into the hypervascularized nidus as seen on the 3D DCE-MRI scan. MWA was performed at $16 \mathrm{~W}$, 
$915 \mathrm{MHz}, 80^{\circ} \mathrm{C}$. Ablation time was dependent on the diameter of the lesion (45-160 s). After ablation, the needle was withdrawn and a sterile dressing was applied.

Pre and post DCE-MRI datasets were used to measure signal intensities (SIs), plasma flow (PF: $\mathrm{ml} / 100 \mathrm{ml} / \mathrm{min}$ ), mean volume of distribution (VD: $\mathrm{ml} / 100 \mathrm{ml}$ ) and mean transit time (MTT: s) using a certified open source software tool for quantitative MRI perfusion analysis (UMMPerfusion, OpossUMM, Germany) (16). In detail, the 3D T1-weighted DCE-MRI data was opened with a 4D viewer of an open source image processing software application (OsiriX version 6.5.1). A region of interest (ROI) was placed within an artery, the plug-in for quantitative MRI perfusion analysis was selected and perfusion analysis was run using the fast-deconvolution algorithm. ROI based measurements for PF, VD and MTT were performed within the OO pre and post MWA.

For long-term clinical follow-up a questionnaire was sent out to all patients after treatment. Questions were asked on a numerical rating scale from 0 to 10 ( 0 being pain-free and 10 being the worst pain imaginable) on the level of pain before and after the procedure, on the current level of pain if any, as well as on how stressful and painful the intervention itself had been perceived.

For statistical analysis, where applicable, $t$-test and Wilcoxon test for paired samples were computed. Means are given with their standard deviation. $p$-Values of 0.05 or less were considered statistically significant. Correlations were computed using Spearman's or Pearson's correlation coefficient. All statistical analyzes were computed using $\mathrm{JMP}^{\circledR}$, Version 13.0 (SAS Institute Inc., Cary, NC, USA).

\section{Results}

Between April 2009 and December 2016, 24 patients (mean age 28 years; nine females) with symptomatic OO were treated with percutaneous, CT-guided MWA. All patients presented with typical pain for a period longer than 1 year managed by nonsteroidal anti-inflammatory drugs and diagnosis was confirmed by initial DCE-MRI. CT-guided MWA was performed with a mean ablation time of $76 \pm 53.26$ $\mathrm{s}($ range $=45-300 \mathrm{~s}$ ). No minor or major adverse events were observed according to the definition of complications by the Society of Interventional Radiology (17) and treatment was technically successful in all cases. OOs were found at the following locations: femoral bone in nine, tibia in eight, and the rib, humerus, acetabulum, fibula, navicular bone of the foot, calcaneus and metatarsal bone in one each. The mean lesion diameter was $5.3 \pm 1.9 \mathrm{~mm}$. Patient characteristics are summarized in Table I.

Out of the 24 patients treated, 22 underwent the 3D DCEMRI protocol as described above. One patient (patient 13) could not be examined with MRI due to claustrophobia, and in one patient of minor age (patient 24), the parents refused MRI imaging. In all of 22 patients examined by DCE-MRI, a hypervascularized lesion was identified, which disappeared on follow-up imaging performed within 2 days of the intervention. In one patient (patient 1), 3D DCE-MRI data could not be evaluated due to severe motion artefacts; pre and post ablation SI measurements however were performed.
DCE-MRI data are summarized in Table II. SI of the lesions $(n=22)$ decreased on average by $89.0 \pm 8.98 \%$ after thermoablation. The SI of adjacent muscle tissue was measured as background signal and remained constant before and after treatment. A Wilcoxon test for paired samples showed significant reduction in lesion SI after ablation $(p<0.0001)$, whereas the difference in muscle SI after ablation remained nonsignificant $(p=0.17)$. Figure 1 shows an exemplary case with SI in DCE-MRI data pre and post ablation.

In the quantitative perfusion analysis of the DCE-MRI data of 21 patients, a paired $t$-test showed no statistically significant change in MTT after ablation $(p=0.54)$, whereas PF $(p=0.0006)$ and VD $(p=0.006)$ decreased significantly.

A Pearson product-moment correlation coefficient ( $r$ ) was computed to assess the relationship between the quantitative perfusion data and the DCE-MRI SI data $(n=21)$. Whereas no positive correlation was found between DCE-MRI SI and MTT, positive correlations were found between DCE-MRI SI and $\mathrm{PF}(\mathrm{r}=0.511, \mathrm{n}=21, p=0.02)$ and DCE-MRI SI and $\mathrm{V}_{\mathrm{D}}$ $(\mathrm{r}=0.4584, \mathrm{n}=21, p=0.04)$. $\mathrm{PF}$ and $\mathrm{V}_{\mathrm{D}}$ were also strongly correlated $(\mathrm{r}=0.9002, \mathrm{n}=21, p<0.0001)$.

All patients except one (patient 1) were pain-free at the 1week follow-up visit. In total, 12 out of 24 patients answered the questionnaire sent to them. The mean follow-up time was $1,082 \pm 691$ days. All patients reported that they felt severely limited in their everyday activities due to the pain experienced from the $\mathrm{OO}$ and perceived the MWA as not or only a little stressful. Ten patients reported that they were free of pain. On a numerical rating scale from 0 to 10 ( 0 being pain-free and 10 being the worst pain imaginable), these patients reported a mean pain level of $6.92 \pm 1.78$ before ablation and of $1.25 \pm 2.22$ after ablation, a difference which was statistically significant $(p<0.0001)$. Of the two patients with persisting pain, one patient had known severe comorbidities and stated that she was not able to differentiate between different pain origins (patient 1). The second patient (patient 15) reported initial subsiding of symptoms 1 week after treatment and 1 year later recurrence of pain to a level of 7 .

The nonparametric Spearman's rank correlation coefficient $\left(r_{s}\right)$ was computed to assess the relationship between pain reduction and MRI data. Only weak correlation was found between level of pain reduction and change in DCE-MRI SI, MTT, PF and VD ( $\mathrm{r}_{\mathrm{s}}=0.11$ to $0.23, \mathrm{n}=12$, all $\left.p>0.05\right)$.

\section{Discussion}

Several studies have shown that DCE-MRI is reliable in the diagnosis of $\mathrm{OO}$ by delineating the hypervascularised nidus of the lesion (8-12). Kalle et al. concluded in their study with 54 patients that $\mathrm{OO}$ can be reliably diagnosed using DCEMRI, especially for exact localization of the nidus (11). In their study with 102 patients, Pottecher et al. found early arterial enhancement of OO on DCE-MRI in $93 \%$ of their 
Table I. Patient characteristics. Maximum lesion diameter was measured on computed tomographic scan. All lesions were ablated at $16 \mathrm{~W}$.

\begin{tabular}{|c|c|c|c|c|c|}
\hline Patient & Age (years) & Gender & Lesion location & Max. lesion diameter (mm) & Ablation time (s) \\
\hline 1 & 46 & $\mathrm{~F}$ & Fourth left dorsal rib & 3 & 60 \\
\hline 2 & 23 & $\mathrm{~F}$ & Right femoral neck & 6 & 60 \\
\hline 3 & 16 & M & Right femoral neck & 4 & 60 \\
\hline 4 & 17 & M & Left calcaneus & 4 & 60 \\
\hline 5 & 17 & M & Left proximal tibia & 3 & 60 \\
\hline 6 & 17 & M & Left femoral neck & 5 & 60 \\
\hline 7 & 31 & M & Right distal tibia & 3 & 60 \\
\hline 8 & 27 & M & Right navicular bone & 3 & 60 \\
\hline 9 & 44 & M & Left femoral neck & 3 & 60 \\
\hline 10 & 34 & $\mathrm{~F}$ & Right distal tibia & 9 & 160 \\
\hline 11 & 47 & $\mathrm{~F}$ & Right 4 th metatarsal bone base & 7 & 60 \\
\hline 12 & 28 & $\mathrm{~F}$ & Right femoral shaft & 3 & 60 \\
\hline 13 & 18 & M & Right proximal humerus & 8 & 60 \\
\hline 14 & 30 & M & Right tibial shaft & 6 & 60 \\
\hline 15 & 32 & M & Right distal tibia & 7 & 45 \\
\hline 16 & 18 & $\mathrm{~F}$ & Right tibial shaft & 3 & 60 \\
\hline 17 & 29 & M & Right lateral condyle of femur & 7 & 120 \\
\hline 18 & 23 & $\mathrm{~F}$ & Right distal femur & 5 & 60 \\
\hline 19 & 7 & M & Left proximal femur & 6 & 60 \\
\hline 20 & 34 & M & Right acetabular roof & 6 & 60 \\
\hline 21 & 46 & M & Left fibula & 5 & 60 \\
\hline 22 & 25 & $\mathrm{~F}$ & Right proximal femur & 8 & 60 \\
\hline 23 & 48 & $\mathrm{~F}$ & Left distal tibia & 7 & 60 \\
\hline 24 & 14 & M & Right tibial shaft & 6 & 60 \\
\hline Mean & 28.0 & - & - & 5.3 & 66.0 \\
\hline
\end{tabular}

patients (9), whereas Liu et al. concluded that DCE-MRI is capable of delineating $\mathrm{OO}$ with a similar reliability to that of CT (8). DCE-MRI allows for functional imaging of the vascularization of the hypervascularized nidus with submillimeter isotropic voxels. Functional delineation of the hypervascularized nidus is important for enabling targeted therapy and post treatment evaluation. Destruction of the capillary channels of an $\mathrm{OO}$ by thermoablation implies concurrent disintegration of nerve endings, which are deemed responsible for the pain experienced by patients (18).

It was demonstrated that MW-based thermoablation leads to a significant post-therapeutic decrease of relative SI of lesions in DCE-MRI (5). Similarly, Teixeira et al. found a statistically significant drop in perfusion of OO measured by DCE-MRI post percutaneous laser therapy (10).

In our study, quantitative perfusion analysis showed a statistically significant decrease in PF and VD of all $\mathrm{OO}$ lesions after percutaneous MWA. Significant changes in MTT were not observed, which might be attributed to the abnormal arterio-venous architecture of OO. Furthermore, positive correlations were found between DCE-MRI SI and PF, as well as $V_{D}$, and $P F$ and $V_{D}$ were also strongly correlated.

In terms of clinical outcome, there was a significant $(p<0.0001)$ reduction in pain described by patients who answered the questionnaire. A weak correlation $\left(\mathrm{r}_{\mathrm{s}}=0.11\right.$ -
Table II. Values for lesion signal intensity (SI), muscle SI, lesion plasma flow (PF), volume of distribution (VD) and (MTT) pre and post ablation. SI of adjacent muscle tissue was taken as background signal in each measurement, and ratios of lesion to muscle tissue SI were calculated.

\begin{tabular}{lccc}
\hline & Pre-ablation & Post-ablation & $p$-Value \\
\hline SI lesion & $146.52 \pm 93.05$ & $52.12 \pm 41.79$ & $<0.0001$ \\
SI muscle & $45.02 \pm 30.38$ & $49.86 \pm 34.30$ & 0.28 \\
SI ratio: lesion/muscle & $4.04 \pm 3.17$ & $1.05 \pm 0.37$ & 0.0001 \\
Lesion PF $(\mathrm{ml} / 100 \mathrm{ml} / \mathrm{min})$ & $229.66 \pm 217.39$ & $46.24 \pm 60.65$ & 0.0006 \\
Lesion V $(\mathrm{ml} / 100 \mathrm{ml})$ & $59.20 \pm 59.23$ & $19.23 \pm 35.60$ & 0.006 \\
Lesion MTT $(\mathrm{s})$ & $16.97 \pm 7.48$ & $15.20 \pm 11.68$ & 0.54 \\
\hline
\end{tabular}

$0.23)$ was found between the level of pain reduction and change in DCE-MRI data post treatment. Patients perceived the MWA itself as not or only a little stressful, underlining the minimally invasive nature of the treatment.

Limitations of our study are firstly the very early conduction of the first follow-up MRI, in most cases only 1 day after the procedure. This was due to organizational reasons, since it would have been difficult to organize the follow-up MRI as an outpatient examination. Furthermore, our follow-up questionnaire was unfortunately answered by 

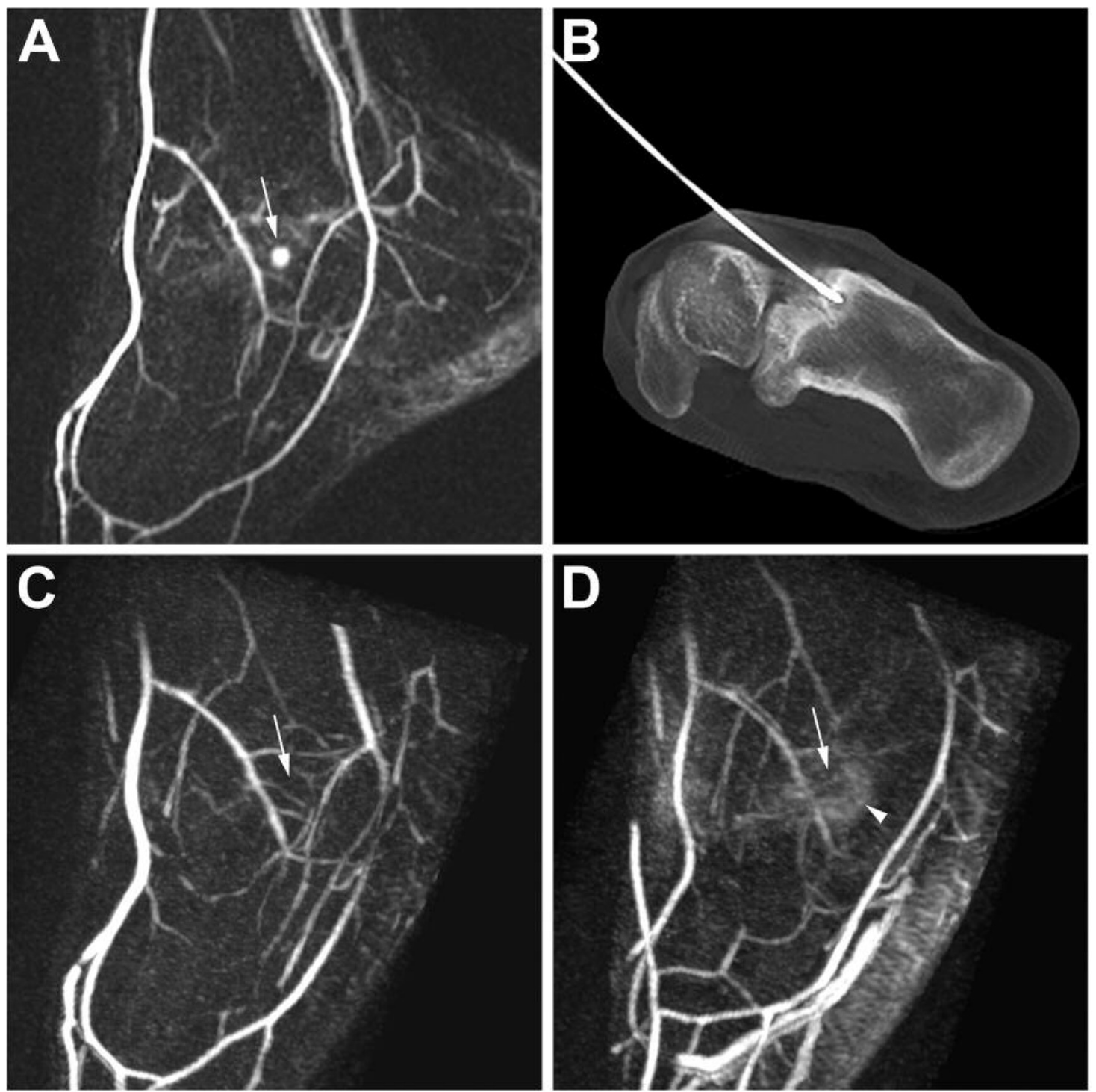

Figure 1. Dynamic contrast-enhanced magnetic resonance imaging (DCE-MRI) and computed tomographic (CT) images of a 17-year-old man with an osteoid osteoma located in the calcaneus. A: Maximum intensity projection (MIP) of pre ablation DCE-MRI data showing a hypervascularized lesion (arrow); B: CT-guided insertion of microwave ablation needle. C: MIP of early (arterial) DCE-MRI data showing absence of the hypervascular lesion (arrow) 1 day after ablation. D: In contrast, MIP of late (venous) DCE-MRI data 1 day after ablation showed perilesional enhancement (arrowhead) corresponding to post ablation inflammation of surrounding tissue.

only about $50 \%(\mathrm{n}=12)$ of our patients, which probably attributed to the only weak correlation found between the level of pain reduction and DCE-MRI data. However, with on average 1,082 days of follow-up, we have a very long follow-up time with stable post ablative results.

In conclusion, in keeping with the available literature, the results of our study demonstrate that 3D DCE-MRI allows for reliable delineation of the hypervascularized $\mathrm{OO}$ nidus and for evaluation of treatment response after percutaneous MWA. MWA is an effective treatment option for $\mathrm{OO}$ with a low-risk profile. Quantitative MRI-perfusion analysis allows for objective treatment evaluation, whereas $\mathrm{PF}$ and $\mathrm{V}_{\mathrm{D}}$ seem to be reliable indicators of successful thermoablation. DCEMRI perfusion analysis may also help to identify early small remnants of perfused OO tissue after MWA.

\section{Conflicts of Interest}

The Authors state no conflict of interest for any aspect of the submitted work. 
The Institute of Clinical Radiology and Nuclear Medicine has research agreements with Siemens Healthineers.

\section{Authors' Contributions}

M.K, N.R. and S.J.D. carried out the interventions. M.K. and T.H. conceived the original idea. M.K. wrote the article with support from T.H., S.J.D., N.R. and S.O.S. S.J.D. and S.O.S. supervised the project.

\section{References}

1 Kransdorf MJ, Stull MA, Gilkey FW and Moser RP Jr.: Osteoid osteoma. Radiographics 11(4): 671-696, 1991. PMID: 1887121. DOI: 10.1148/radiographics.11.4.1887121

2 Volkmer D, Sichlau M and Rapp TB: The use of radiofrequency ablation in the treatment of musculoskeletal tumors. J Am Acad Orthop Surg 17(12): 737-743, 2009. PMID: 19948698.

3 Motamedi D, Learch TJ, Ishimitsu DN, Motamedi K, Katz MD, Brien EW and Menendez L: Thermal ablation of osteoid osteoma: Overview and step-by-step guide. Radiographics 29(7): 2127-2141, 2009. PMID: 19926767. DOI: 10.1148/rg. 297095081

4 Filippiadis DK, Velonakis G, Kostantos C, Kouloulias V, Brountzos E, Kelekis N and Kelekis A: Computed tomographyguided radiofrequency ablation of intra-articular osteoid osteoma: A single centre's experience. Int J Hyperthermia 33(6): 670-674, 2017. PMID: 28540776. DOI: 10.1080/02656736. 2017.1294711

5 Kostrzewa M, Diezler P, Michaely H, Rathmann N, Attenberger UI, Schoenberg SO and Diehl SJ: Microwave ablation of osteoid osteomas using dynamic MR imaging for early treatment assessment: Preliminary experience. J Vasc Interv Radiol 25(1): 106-111, 2014. PMID: 24290095. DOI: 10.1016/j.jvir.2013. 09.009

6 Basile A, Failla G, Reforgiato A, Scavone G, Mundo E, Messina M, Caltabiano G, Arena F, Ricceri V, Scavone A and Masala S: The use of microwaves ablation in the treatment of epiphyseal osteoid osteomas. Cardiovasc Intervent Radiol 37(3): 737-742, 2014. PMID: 23989501. DOI: 10.1007/s00270-013-0722-z

7 Rinzler ES, Shivaram GM, Shaw DW, Monroe EJ and Koo KSH: Microwave ablation of osteoid osteoma: Initial experience and efficacy. Pediatr Radiol, 2019. PMID: 30617514. DOI: 10.1007/s00247-018-4327-1

8 Liu PT, Chivers FS, Roberts CC, Schultz CJ and Beauchamp CP: Imaging of osteoid osteoma with dynamic gadoliniumenhanced MR imaging. Radiology 227(3): 691-700, 2003. PMID: 12773675. DOI: 10.1148/radiol.2273020111

9 Pottecher P, Sibileau E, Aho S, Hamze B, Parlier C, Laredo JD and Bousson V: Dynamic contrast-enhanced $\mathrm{mr}$ imaging in osteoid osteoma: Relationships with clinical and CT characteristics. Skeletal Radiol 46(7): 935-948, 2017. PMID: 28401265. DOI: $10.1007 / \mathrm{s} 00256-017-2645-2$
10 Teixeira PA, Chanson A, Beaumont M, Lecocq S, Louis M, Marie B, Sirveaux F and Blum A: Dynamic MR imaging of osteoid osteomas: Correlation of semiquantitative and quantitative perfusion parameters with patient symptoms and treatment outcome. Eur Radiol 23(9): 2602-2611, 2013. PMID: 23695220. DOI: $10.1007 / \mathrm{s} 00330-013-2867-1$

11 von Kalle T, Langendorfer M, Fernandez FF and Winkler P: Combined dynamic contrast-enhancement and serial 3Dsubtraction analysis in magnetic resonance imaging of osteoid osteomas. Eur Radiol 19(10): 2508-2517, 2009. PMID: 19444454. DOI: 10.1007/s00330-009-1430-6

12 Zampa V, Bargellini I, Ortori S, Faggioni L, Cioni R and Bartolozzi C: Osteoid osteoma in atypical locations: The added value of dynamic gadolinium-enhanced MR imaging. Eur J Radiol 71(3): 527-535, 2009. PMID: 18599238. DOI: 10.1016/ j.ejrad.2008.05.010

13 Riffel P, Haneder S, Attenberger UI, Brade J, Schoenberg SO and Michaely HJ: Combined large field-of-view MRA and timeresolved MRA of the lower extremities: Impact of acquisition order on image quality. Eur J Radiol 81(10): 2754-2758, 2012. PMID: 22185939. DOI: 10.1016/j.ejrad.2011.12.003

14 Attenberger UI, Haneder S, Morelli JN, Diehl SJ, Schoenberg SO and Michaely HJ: Peripheral arterial occlusive disease: Evaluation of a high spatial and temporal resolution 3-T MR protocol with a low total dose of gadolinium versus conventional angiography. Radiology 257(3): 879-887, 2010. PMID: 20959539. DOI: 10.1148/radiol.10100781

15 Voth M, Haneder S, Huck K, Gutfleisch A, Schonberg SO and Michaely HJ: Peripheral magnetic resonance angiography with continuous table movement in combination with high spatial and temporal resolution time-resolved MRA with a total single dose $(0.1 \mathrm{mmol} / \mathrm{kg})$ of gadobutrol at 3.0 T. Invest Radiol 44(9): 627633, 2009. PMID: 19652610. DOI: 10.1097/RLI.0b0 $13 \mathrm{e} 3181 \mathrm{~b} 4 \mathrm{c} 26 \mathrm{c}$

16 Zollner FG, Weisser G, Reich M, Kaiser S, Schoenberg SO, Sourbron SP and Schad LR: Ummperfusion: An open source software tool towards quantitative MRI perfusion analysis in clinical routine. J Digit Imaging 26(2): 344-352, 2013. PMID: 22832894. DOI: 10.1007/s10278-012-9510-6

17 Callstrom MR, York JD, Gaba RC, Gemmete JJ, Gervais DA, Millward SF, Brown DB, Dupuy D, Goldberg SN, Kundu S, Rose SC, Thomas JJ and Cardella JF: Research reporting standards for image-guided ablation of bone and softtissue tumors. J Vasc Interv Radiol 20(12): 1527-1540, 2009. PMID: 19864161. DOI: 10.1016/j.jvir.2009.08.009

18 Hasegawa T, Hirose T, Sakamoto R, Seki K, Ikata T and Hizawa $\mathrm{K}$ : Mechanism of pain in osteoid osteomas: An immunohistochemical study. Histopathology 22(5): 487-491, 1993. PMID: 8344659.
Received May 2, 2019

Revised May 14, 2019

Accepted May 16, 2019 